\title{
L'infection émergente à Haemophilus influenzae de sérotype a et un possible vaccin : Science de la mise en œuvre
}

\author{
L Barreto', AD Cox ${ }^{1}$, M Ulanova², MG Bruce ${ }^{3}$, RSW Tsang ${ }^{4 *}$
}

\section{Résumé}

Haemophilus influenzae de sérotype b (Hib) était l'une des principales causes de méningite chez les enfants, jusqu'à ce qu'un vaccin conjugué contre Hib soit intégré au programme de vaccination systématique des nourrissons et que l'infection causée par Hib chez l'enfant ne soit presque éliminée. En Alaska, dans le Nord du Canada ainsi que dans d'autres pays où vivent des peuples autochtones, $H$. influenzae de sérotype a (Hia) est devenue une cause importante de pneumonie, de méningite et d'arthrite septique, en particulier chez les enfants de moins de 24 mois. Une initiative gouvernementale conjointe entre l'Agence de la santé publique du Canada (ASPC) et le Conseil national de recherches du Canada (CNRC) a été menée afin de déterminer si un vaccin contre Hia pourrait être mis au point pour le bien commun.

L'initiative comprenait des partenariats stratégiques avec des chercheurs cliniciens établis à Thunder Bay (Ontario) fournissant des services de santé aux Autochtones, et avec un programme établi en Alaska qui a pour but d'étudier et de promouvoir la santé en Arctique (l'Arctic Investigations Program). Ce programme est une branche des centres pour le contrôle et préventions des maladies (Centers for Disease Control and Prevention, CDC) aux États-Unis. Cette étude, financée et entreprise par le gouvernement, a révélé qu'il était possible de mettre au point un vaccin contre le Hia et qu'il était essentiel d'assurer une surveillance continue incluant la caractérisation des différentes souches bactériennes afin de connaître le risque de propagation de Hia en Amérique du Nord et dans le monde entier.

\author{
Affiliations \\ ${ }^{1}$ Portefeuille des Thérapeutiques \\ en santé humaine, Conseil \\ national de recherches du \\ Canada, Ottawa (Ontario) \\ ${ }^{2}$ École de médecine du Nord de \\ I'Ontario, Université Lakehead, \\ Thunder Bay (Ontario) \\ ${ }^{3}$ Arctic Investigations Program, \\ Division of Preparedness and \\ Emerging Infections, Centers for \\ Disease Control and Prevention \\ (CDC), Anchorage (Alaska) \\ ${ }^{4}$ Laboratoire national de \\ microbiologie, Agence de la santé \\ publique du Canada, Winnipeg \\ (Manitoba)
}

*Correspondance : raymond.
tsang@phac-aspc.gc.ca

Citation proposée : Barreto L, Cox AD, Ulanova M, Bruce MG, Tsang RSW. L'infection émergente à Haemophilus influenzae de sérotype a et un possible vaccin : Science de la mise en œuvre. Relevé des maladies transmissibles au Canada. 2017;43(5):94-7. https: //doi.org/10.14745/ccdr.v43i05a01f

\section{Introduction}

La science de la mise en œuvre illustre parfaitement la raison d'être de la recherche en santé : obtenir une bonne compréhension des différents phénomènes, tester des hypothèses, élaborer des solutions et évaluer efficacité des solutions découvertes afin d'améliorer la santé des individus et de la population. Toutefois, ce processus est rarement aussi simple qu'il n'y paraît. Les actes de l'atelier sur Haemophilus influenzae de sérotype a (Hia) figurant dans ce numéro du Relevé des maladies transmissibles au Canada (1) ont permis d'identifier les différents types de données probantes nécessaires à la mise au point d'un nouveau vaccin ainsi que les défis qui restent à relever afin de convertir les recherches initiales en un produit approuvé. Dans le présent éditorial, nous expliquerons ce que constitue Hia et son émergence, nous mettrons aussi en évidence certains des aspects uniques sur la mise au point d'un vaccin contre Hia et soulignerons l'importance d'une surveillance continue afin d'observer les tendances des infections causées par cette bactérie, tant en Amérique du Nord que dans le reste du monde.

\section{Contexte}

Haemophilus influenzae $(H$. influenzae) est un agent pathogène humain qui se loge habituellement dans les voies respiratoires supérieures, mais que l'on retrouve parfois dans la muqueuse urogénitale, causant des infections urinaires, néonatales et obstétricales (2-4). Les souches de H. influenzae comprennent celles qui présentent à leur surface des capsules consitituées de polysaccharidiques qui subdivisent les souches sur le plan antigénique en six sérotypes capsulaires différents (de a à f), et celles qui sont exemptes de capsules (appelées non typables) (5). Les souches capsulées ont tendance à causer des maladies invasives comme la méningite, la septicémie, la pneumonie bactériémique et l'arthrite septique, tandis que les souches non capsulées provoquent généralement des infections non invasives comme l'otite moyenne, la sinusite et la bronchite.

Hib était l'une des principales causes de méningite chez les enfants de moins de cinq ans $(6,7)$ jusqu'à ce qu'un vaccin conjugué contre cette souche ne soit intégré au programme de vaccination systématique des nourrissons au début des années 1990. Depuis lors, les infection causées par le Hib chez les enfants ont pratiquement été éliminées (8). 
Malheureusement, les taux décroissants d'infection causées par Hib observés à la suite de la vaccination des nourrissons n'ont pas mené à l'élimination complète de l'infection invasive casuée par $H$. influenzae. À la suite de l'introduction du vaccin contre Hib, des souches de $H$. influenzae de sérotype non-b et non typables sont devenues plus courantes (9-11). En Alaska (États-Unis) (12) et dans le Nord du Canada (13-15), ainsi que dans des régions où vivent en grandes proportions le peuple autochtone $(16,17)$, Hia est devenue une cause importante de maladies invasives, en particulier chez les enfants de moins de 24 mois $(18,19)$. La souche Hia provoque des maladies telles que la pneumonie, la méningite et l'arthrite septique, et est associée à des taux très élevés de morbidité et de mortalité, ainsi qu'à de très nombreux cas d'invalidité à vie $(12,13,18)$.

\section{Aspects uniques de la mise au point d'un vaccin contre Hia}

Dans le présent numéro du Relevé des maladies transmissibles au Canada, Cox et collègues ont identifié les données probantes nécessaires à la mise au point d'un nouveau vaccin. Ces données probantes sont constituées : de données de surveillance, de recherches en laboratoire, d'études précliniques, de considérations réglementaires, de bonnes pratiques de fabrication dans la production de lots de vaccins aux fins d'essais cliniques, de capacité à mener des essais cliniques, de pratiques exemplaires en matière de partenariats public-privé et bien plus encore (20). Les actes de conférence mettent en relief deux aspects uniques liés à la mise au point du vaccin contre Hia. Tout d'abord, ce projet est né d'une collaboration interministérielle au sein du gouvernement du Canada faisant intervenir des partenariats stratégiques, ensuite, il s'agit d'une initiative de recherche gouvernementale avec des fonds internes affectés à des études de validation de principe et à la surveillance continue.

\section{Collaboration interministérielle}

Au début des années 2000, l'Agence de la santé publique du Canada (ASPC) et le Conseil national de recherches du Canada (CNRC) ont conjugué leurs efforts afin de collaborer à «l'expansion de la mise au point de vaccins au Canada ». Ce partenariat s'est formé sur la base des capacités et de l'expertise uniques de deux ministères différents afin de mettre en œuvre des solutions scientifiques en faveur du bien commun. À cette époque, les activités de surveillance en laboratoire au Laboratoire national de microbiologie (LNM) avaient permis d'identifier un pourcentage élevé d'isolats de sérotype a responsables d'infections invasives causées par $\mathrm{H}$. Influenzae (20). La consultation du Conseil des médecins hygiénistes provinciaux et territoriaux ainsi que l'examen subséquent des données sur l'infection invasive causée par Hia provenant des territoires du Nord du Canada et collectées au moyen du Système international de surveillance circumpolaire ont confirmé que la souche Hia était un important agent pathogène émergent $(21,22)$ causant de graves maladies invasives et contre lequel il n'existait actuellement aucun vaccin. S'inspirant de la réussite du vaccin conjugué contre le Hib mis au point dans les années 1980 afin de lutter contre l'infection invasive contre le Hib, des similarités sur le plan biologique entre Hia et Hib et les maladies qu'elles causent, des travaux de recherche ont été lancés afin de jeter les bases de la mise au point d'un vaccin conjugué contre Hia.

\section{Partenariats stratégiques externes}

Le campus de Thunder Bay de l'École de médecine du Nord de I'Ontario à l'Université Lakehead est situé dans le Nord-Ouest de l'Ontario, et ses activités d'enseignement et de recherche sont rattachées au Centre régional des sciences de la santé de Thunder Bay — un hôpital universitaire de 375 lits. L'École de médecine du Nord de l'Ontario et l'hôpital universitaire fournissent tous deux divers services de santé à une population comptant une proportion élevée d'Autochtones. Du côté des États-Unis, le L'Arctic Investigations Program (AIP) des Centers for Disease Control and Prevention (CDC) situés à Anchorage, en Alaska, possèdent une vaste expérience sur l'étude de l'infection invasive causée par Hib. Cette infection a débuté au début des années 1970, avant la mise au point du vaccin conjugué contre Hib. Les chercheurs de I'AIP avaient également mené des essais cliniques sur le vaccin conjugué contre Hib, conduisant finalement à la découverte de l'actuel vaccin conjugué Hib-OMV (PRP-OMP) spécialement mis au point pour les enfants autochtones américains (23).

Chaque partenaire de cette collaboration multidisciplinaire apporte au groupe une expertise unique, mais complémentaire. Le LNM de I'ASPC et I'AIP des CDC américaines, effectuent une surveillance en laboratoire des maladies infectieuses dans leur pays respectif et le LNM possède une vaste souchothèque conservée dans une biobanque à des fins de recherche et développement. Le CNRC s'appuie sur une longue tradition de recherches sur les glycoprotéines microbiennes, possède une vaste expérience dans le domaine des technologies de conjugaison de glycoprotéines et a mobilisé l'industrie afin de mettre au point des vaccins. L'École de médecine du Nord de l'Ontario a accès à une grande installation clinique régionale et possède une expertise unique en immunologie des maladies infectieuses ainsi qu'en essais immunologiques appliqué au domaine des vaccins. La formation de ce partenariat vise à favoriser la réussite de la mise au point d'un vaccin conjugué contre Hia.

\section{Travaux de surveillance et de mise au point de vaccin financés par le gouvernement}

L'autre aspect unique de la mise au point d'un vaccin contre Hia réside dans le fait qu'il s'agit d'une initiative gouvernementale. Le LNM de I'ASPC a fait état de l'éventuelle nécessité de mettre au point un vaccin contre cette souche grâce à son programme de surveillance en laboratoire financé par le gouvernement du Canada. Le scientifique responsable de ce dossier au LNM possède des connaissances sur les vaccins bactériens ainsi que sur les relations professionnelles avec les scientifiques du CNRC, ce qui a ouvert la voie à des possibilités de collaboration. Un petit investissement du gouvernement du Canada a permis aux chercheurs du CNRC de purifier le polysaccharide capsulaire de la souche Hia et d'obtenir la réaction de conjugaison requise pour produire un lot de recherche du vaccin conjugué contre Hia. Les études d'immunogénicité menées sur des animaux de laboratoire ont confirmé son efficacité à induire des anticorps bactéricides, ce qui constitue un marqueur de substitution reconnu de l'immunité protectrice contre $H$. influenzae. Ainsi, 
les études réalisées à ce jour ont permis d'établir la validation de principe préclinique selon laquelle l'approche utilisée pour mettre au point un vaccin conjugué contre Hia devrait être aussi efficace que celle utilisée pour le vaccin conjugué contre Hib.

La surveillance épidémiologique actuelle a révélé que les populations les plus à risque de contracter des infections invasives causées par Hia sont les enfants autochtones de moins de cinq ans et les adultes autochtones immunodéprimés vivant en Amérique du Nord ainsi que dans d'autres régions. De nombreux efforts ont été consacrés à la consultation des communautés les plus touchées. Au Canada, deux ateliers (y compris celui dont les actes de conférence sont publiés dans le présent numéro du Relevé des maladies transmissibles au Canada) ont été organisés réunissant des membres des communautés autochtones et des chercheurs de l'École de médecine du Nord de l'Ontario qui continuent de faire participer ces communautés à leur recherche.

\section{Conclusion}

Utiliser les données probantes pour orienter la pratique est une entreprise complexe. En ce qui concerne la mise au point de vaccins, les données probantes visant à démontrer le fardeau de la maladie, les études en laboratoire ainsi que les consultations auprès des intervenants en santé publique, les personnes touchées par la maladie, les experts en matière de réglementation et les partenaires de l'industrie sont des composantes essentielles au processus. Toutefois, dans le cas du vaccin contre Hia, deux éléments uniques et additionnels sont également importants : le fait que le financement et la recherche gouvernementale a permis d'identifier le problème, et qu'un partenariat gouvernemental a mené à une validation de principe pour un vaccin, ce qui a favorisé la mobilisation d'autres intervenants afin de faire avancer la mise au point de cette solution. En raison de la nature évolutive des agents infectieux, l'épidémiologie de l'infection invasive causée par $H$. influenzae continuera probablement à changer. Une surveillance continue incluant la caractérisation des souches est donc primordiale pour suivre la propagation potentielle de Hia en Amérique du Nord et dans le monde entier et, peut-être ultérieurement, documenter sa disparition grâce à l'introduction d'un nouveau vaccin.

\section{Déclaration des auteurs}

Tous les auteurs (L.B., A.D.C., M.U., M.G.B. et R.S.W.T.) ont participé à l'élaboration de cet éditorial. L.B. et R.S.W.T. ont préparé la première ébauche et tous les autres auteurs ont contribué à la version finale en ajoutant des commentaires et des suggestions.

\section{Avis de non-responsabilité}

Les constatations et conclusions exprimées dans cet article n'engagent que leurs auteurs et ne reflètent pas nécessairement la position officielle de l'Agence de la santé publique du Canada ni des Centers for Disease Control and Prevention des États-Unis.

\section{Conflit d'intérêts}

A.D.C., M.G.B. et R.S.W.T. n'ont aucun conflit d'intérêts à déclarer.

M.U. bénéficie d'une subvention de recherche libre octroyée par Pfizer pour une étude indépendante. L.B. est conseiller scientifique principal auprès de Inventprise/InventVac, Redmond (État de Washington)/Vancouver (Colombie-Britannique), et conseiller stratégique auprès NÉOMED-LABS, de Montréal (Québec). Il a travaillé au sein du groupe Vaccins et immunothérapeutiques (Thérapeutiques en santé humaine) du Conseil national de recherches du Canada (CNRC) de 2011 à 2015, et agi à titre de consultant auprès du CNRC pour la mise au point d'un vaccin anti-Hia. II a travaillé avec Sanofi Pasteur de 1988 à 2010 à la mise au point d'un vaccin combiné anti-Hib.

\section{Remerciements}

Nous remercions le Dr Rainer Engelhardt, le Dr John Spika de I'ASPC et le D' Jim Richards du CNR pour la mise en œuvre de la phase initiale de ce projet, le $D^{r}$ Thomas Hennessy et la

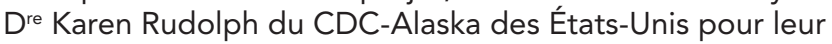
soutien continu, le Dr William McCready et le Dr Eli Nix de l'École de médecine du Nord de l'Ontario, le Dr Lakshmi Krishnan, le Dr Wei Zou, Frank St. Michael, Chantelle Cairns, Dean Williams et Rhonda Kuo Lee du CNR pour leur soutien au projet sur la souche Hia et la Dre Patricia Huston pour avoir suggéré la structure de cet article.

\section{Références}

1. Cox A, Barreto L, Ulanova M, Bruce MG, Tsang RSW, au nom des collaborateurs à la conférence. Développement d'un vaccin contre Haemophilus influenzae de sérotype a : Compte-rendu d'un atelier. Relevé des maladies transmissibles au Canada. 2017;43(5):98-105. Disponible à l'adresse : http://www.phac-aspc.gc.ca/publicat/ccdrrmtc/17vol43/dr-rm43-05/ar-2-fra.php.

2. Slack MPE. Haemophilus. In: Borriello SP, Murray PR, Funke $G$ eds. Topley and Wilson's microbiology and microbial infections, 10th ed. Vol 2. Bacteriology. London, UK: Edward Arnold Ltd.; 2006. p.1692-1718.

3. Gabre-Kidan T, Lipsky BA, Plorde JJ. Haemophilus influenzae as a cause of urinary tract infections in men. Arch Inter Med. 1984;144:1623-1627.

4. Wallace Jr. RJ, Baker CJ, Quintones FJ, Hollis DG, Weaver RE, Wiss K. Nontypable Haemophilus influenzae (biotype 4) as a neonatal, maternal, and genital pathogen. Rev Infect Dis. 1983;5:123-36.

5. Pittman M. Variation and type specificity in the bacterial species Haemophilus influezae. J Exp Med. 1931;53:471-92.

6. Shapir ED, Ward JJ. The epidemiology and prevention of disease caused by Haemophilus influenzae type b. Epidemiol Rev 1991;13:113-142. 
7. Ulanova M, Tsang RSW. Invasive Haemophilus influenzae disease: Changing epidemiology and host-parasite interactions in the 21st century. Infect Gen Evol. 2009;9:594605.

8. Bath S, Bisgard K, Murphy T, Shutt K, Rosenstein N, Ohuabunwo C, and the Centers for Disease Control and Prevention (CDC). Progress towards elimination of Haemophilus influenzae type $b$ invasive disease among infants and children---United States, 1998-2000. MMWR Morb Mortal Wkly Rep. 2002;51:234-7.

9. Urwin G, Krohn JA, Deaver-Robinson K, Wenger JD, Farley MM, and the Haemophilus influenzae Study Group. Invasive disease due to Haemophilus influenzae serotype f: clinical and epidemiologic characteristics in the $\mathrm{H}$. influenzae serotype b vaccine era. Clin Infect Dis. 1996;22:1069-76.

10. Sadeghi-Aval P, Tsang RSW, Jamieson F, Ulanova M. Emergence of non-type $b$ encapsulated Haemophilus influenzae as a cause of pediatric meningitis in Northwestern Ontario. Can J Infect Dis Med Microbiol. 2013;24:13-6.

11. Van Eldere J, Slack MPE, Ladhani S, Cripps AW. Nontypeable Haemophilus influenzae, an under-recognised pathogen. Lancet Infect Dis. 2014;14:1281-92.

12. Bruce MG, Zulz T, DeByle $C$, Singleton R, Hurlburt D, Bruden $D$, et al. Haemophilus influenzae serotype a invasive disease, Alaska, USA, 1983-2011. Emerg Infect Dis. 2013;19:932-7.

13. Boisvert AA, Moore D. Invasive disease due to Haemophilus influenzae type a in children in Canada's north: A priority for prevention. Can J Infect Dis Med Microbiol. 2015;26:291-2.

14. Tsang RSW, Li YA, Mullen A, Baikie M, Whyte K, Shuel M, et al. Laboratory characterization of invasive Haemophilus influenzae isolates from Nunavut, Canada, 2000-2012. Int J Circumpolar Health. 2016;75:297-8.

15. Bruce MG, Deeks SL, Zulz T, Navarro C, Palacios C, Cases CC, et al. Epidemiology of Haemophilus influenzae serotype a, North American Arctic, 2000-2005. Emerg Infect Dis. 2008;14:48-55.
16. Millar EV, O'Brien KL, Watt JP et al. Epidemiology of invasive Haemophilus influenzae type a disease among Navajo and White Mountain Apache children, 1988-2003. Clin Infect Dis. 2005;40:823-30.

17. Kelly L, Tsang RSW, Morgan A, Jamieson FB, Ulanova M. Invasive disease caused by Haemophilus influenzae type a in Northern Ontario First Nations communities. J Med Microbiol. 2011;60:384-90.

18. Ulanova M, Tsang RSW. Haemophilus influenzae serotype a as a cause of serious invasive infections. Lancet Infect Dis 2014; 14: 70-82.

19. Tsang RSW, Bruce MG, Lem M, Barreto L, Ulanova M. A review of invasive Haemophilus influenzae disease in the Indigenous populations in North America. Epidemiol Infect. 2014; 142:1344-54.

20. Tsang RSW, Mubareka S, Sill ML, Wylie J, Skinner S, Law DKS. Invasive Haemophilus influenzae in Manitoba, Canada, in the postvaccination era. J Clin Microbiol. 2006;44:1530-5.

21. Bruce MG, Deeks SL, Zulz T, Navarro C, Palacios C, Cases CC, et al. Epidemiology of Haemophilus influenzae serotype a, North American Arctic, 2000-2005. Emerg Infect Dis. 2008;14:48-55.

22. Desai S, Lee YA, Zulz T, Bruce M. Surveillance des maladies bactériennes invasives dans l'Arctique nord-américain dans le cadre du projet de Surveillance circumpolaire internationale. Relevé des maladies transmissibles au Canada. 2015;41(3):55-8. Disponible à l'adresse : http:// www.phac-aspc.gc.ca/publicat/ccdr-rmtc/15vol41/drrm41-03/surv-2-fra.php.

23. American Academy of Pediatrics. Summary of infectious diseases, Section 3, Haemophilus influenzae infections, pp. 345-352. In: Pickering LK, Baker CJ, Kimberlin DW, Long SS, eds. Red Book: 2010 Report of the Committee on Infectious Diseases. Elk Groove Village, IL: American Academic of Pediatrics; 2012. 